\title{
Chemical Exchange Saturation Transfer Contrast Agents for Magnetic Resonance Imaging
}

\author{
A. Dean Sherry ${ }^{1,2}$ and Mark Woods ${ }^{1}$ \\ ${ }^{1}$ Department of Chemistry, University of Texas at Dallas, Richardson, Texas 75080; email: \\ sherry@utdallas.edu \\ ${ }^{2}$ Advanced Imaging Research Center, University of Texas Southwestern Medical Center, Dallas, \\ Texas 75390-8568; email: dean.sherry@utsouthwestern.edu
}

\section{Abstract}

Magnetic resonance imaging (MRI) contrast agents have become an important tool in clinical medicine. The most common agents are $\mathrm{Gd}^{3+}$-based complexes that shorten bulk water $\mathrm{T}_{1}$ by rapid exchange of a single innersphere water molecule with bulk solvent water. Current gadolinium agents lack tissue specificity and typically do not respond to their chemical environment. Recently, it has been demonstrated that MR contrast may be altered by an entirely different mechanism based on chemical exchange saturation transfer (CEST). CEST contrast can originate from exchange of endogenous amide or hydroxyl protons or from exchangeable sites on exogenous CEST agents. This has opened the door for the discovery of new classes of responsive agents ranging from MR gene reporter molecules to small molecules that sense their tissue environment and respond to biological events.

\section{Keywords}

MRI contrast agents; CEST; PARACEST

\section{MAGNETIC RESONANCE IMAGING CONTRAST AGENTS: HISTORICAL ASPECTS}

Paramagnetic complexes of $\mathrm{Gd}^{3+}$ are now widely used as magnetic resonance imaging (MRI) contrast agents in clinical medicine. These low-molecular-weight $\mathrm{Gd}^{3+}$ complexes alter image contrast via changes in the spin-lattice relaxation rate $\left(\mathrm{T}_{1}\right)$ of tissue water, which translates to brighter intensities in $\mathrm{T}_{1}$ weighted images. The physical properties of $\mathrm{Gd}^{3+}$-based contrast agents have been thoroughly studied in recent years and the mechanisms that determine the efficiency of such agents are reasonably well understood $(1,2)$. These detailed studies have formed the basis of numerous efforts to create responsive or smart MRI agents: $\mathrm{Gd}^{3+}$-based agents that respond to changes in $\mathrm{pH}(3-9) ; \mathrm{Zn}^{2+}$ and $\mathrm{Ca}^{2+}$ ion concentrations (10-12), a variety of anions $(13,14)$, enzymes (15-17), and proteins (18) have been reported. Although new $\mathrm{Gd}^{3+}$-based agents continue to be widely investigated, they do have some limitations as a platform for responsive MRI agents. The effectiveness of a $\mathrm{Gd}^{3+}$ contrast agent is measured in terms of its relaxivity, defined as the increase in water proton relaxation rate per unit concentration of contrast agent (per millimolar per second). The relaxivity of a complex is

Copyright $\odot 2008$ by Annual Reviews. All rights reserved DISCLOSURE STATEMENT

The authors are not aware of any biases that might be perceived as affecting the objectivity of this review. 
dependent on multiple physical constants, including the magnetic properties of the $\mathrm{Gd}^{3+}$ complex, constants related to electron-spin relaxation, rotational mobility, and the number of metal-bound water molecules $(q)(1,2)$. The net result is that all $\mathrm{Gd}^{3+}$ complexes relax water to some extent and their presence always affects what is observed in the MR image. So, even though the off form of a responsive $\mathrm{Gd}^{3+}$ agent affects an MR image less than the on form, it continues to have some effect. It is therefore necessary to account for the concentration of the agent if these $\mathrm{Gd}^{3+}$-based agents are used in vivo $(19,20)$.

\section{THE BASICS OF CHEMICAL EXCHANGE SATURATION TRANSFER}

$\mathrm{Gd}^{3+}$ agents are widely used in clinical imaging and have been successful in that setting. However, their limitations and drawbacks, particularly in association with responsive agents, suggest that an alternative mechanism for generating image contrast may be able to provide additional information that may improve diagnosis by MRI. Chemical exchange saturation transfer, or CEST, is a technique that has been used in nuclear magnetic resonance (NMR) for more than four decades and is now generating increasing interest as a means of generating contrast in MR images.

The phenomenon of chemical exchange has been studied by NMR spectroscopy for many years. In 1957, McConnell \& Thompson (21) modified the NMR Bloch equations to include terms for chemical exchange and reported proton transfer rates between the ammonium ion and water (22). But the first example of a CEST experiment was performed by Forsén \& Hoffman (23), who used the method to measure proton transfer rates between salicylaldehyde and water. This CEST technique is also referred to as saturation transfer (ST) or magnetization transfer (MT) in some publications. Nevertheless, general methods such as this have been widely applied in both chemistry and biology to determine unidirectional rate constants of simple reactions for many years.

To have CEST, the system must be in chemical exchange. These chemical exchange processes might be exchange of the $\gamma$-phosphate of ATP and the phosphate group of creatine phosphate (PCr) catalyzed by the enzyme creatine kinase, the two methyl groups attached to a nitrogen atom in an asymmetrical molecule that exchange their spatial positions via nitrogen inversion, or, for the purposes of the current discussion, an exchangeable proton of a molecule that exchanges with those of the solvent water. Consider two general exchanging pools, A and B, perturbed by application of a saturation pulse to pool B (Figure 1). Saturation is the process by which the Boltzmann distribution of nuclear spins, aligned either with or against the magnetic field, is temporarily altered by applying a soft (low-power) irradiation pulse at the resonance frequency of a nucleus of interest. The number of spins aligned against the field is increased at the expense of those aligned with the field (Figure 1). Once the number of spins aligned with and against the field is equal, the system is said to be saturated, the net magnetization is zero, and no signal is observed in the NMR spectrum. The nuclei in Pool A are in exchange with the saturated nuclei in Pool B. This exchange transfers spins that are aligned against the field from Pool B to Pool A and spins aligned with the field from Pool A to Pool B. The net result is transfer of saturation from Pool B to Pool A and a concomitant decrease in the signal intensity of the nuclei in Pool A (Figure 1). Longitudinal relaxation returns each system to its equilibrium distribution of spins, so eventually the system reaches steady state. The steady-state intensity $\left(\mathrm{M}_{\mathrm{Aoc}} / \mathrm{M}_{\mathrm{A} 0}\right)$ of the nuclei in Pool $\mathrm{A}$ is given by Equation 1. We refer readers interested in a more in-depth description of the mechanism of CEST to an earlier review (24). 


$$
\frac{M_{A \infty}}{M_{A 0}}=\frac{1}{\left(1+k_{2} T_{1 B}\right)}
$$

The model presented in Figure 1 also implies that the chemical shift of the two exchanging pools must differ substantially. For diamagnetic compounds, the chemical shift difference $(\Delta \omega)$ between exchangeable protons and bulk water is typically $<5 \mathrm{ppm}$, which corresponds to $315 \mathrm{~Hz}$ at $1.5 \mathrm{~T}$ and $630 \mathrm{~Hz}$ at 3T. Assuming the slow-to-intermediate exchange condition $\left(\mathrm{k}_{\mathrm{ex}} \leq \Delta \omega\right)$ represents a rough boundary condition for CEST, this means that exchange must be slower than $\sim 1980 \mathrm{~s}^{-1}$ using a 1.5T scanner or slower than $\sim 3960 \mathrm{~s}^{-1}$ using a 3T scanner to observe CEST As $\Delta \omega$ is small in diamagnetic systems, the applied presaturation frequency must by definition be close to that of solvent water so partial direct off-resonance saturation of the solvent water can occur (25). This problem is accentuated in vivo where the bulk water signal tends to be broader. To extract the effects of a diamagnetic CEST agent then, whether it is an endogenous compound or an exogenous agent, it is often necessary to subtract the effects of direct off-resonance saturation by measuring the effect of presaturation at equal frequency offset difference on each side of the central bulk water peak and report the difference as an asymmetry ratio (26). In an imaging sense, one collects two images, one after presaturation at the exchange site of interest and another at an equivalent frequency offset on the opposite side of bulk water. The difference between these two image intensities should then report only the effects of the exchanging CEST agent. Although the CEST effect generated from diamagnetic endogenous CEST agents often does not differ much from that due to direct off-resonance saturation of bulk water, it is nonetheless large enough to be useful in a number of applications.

\section{ENDOGENOUS CHEMICAL EXCHANGE SATURATION TRANSFER}

Because the effect of CEST is to reduce magnetization in Pool A, the bulk water pool, the net result is a loss of signal intensity and a darkening of the image, or negative contrast. This is in contrast to $\mathrm{Gd}^{3+}$ agents, which typically serve as positive contrast agents by brightening images. As a general rule, positive contrast is preferable simply because the human eye can more easily discriminate a slight increase in brightness than a slight increase in darkness. The image darkening effect of adding a presaturation magnetization transfer pulse has proven useful, however, in a number of applications. One such example is time-of-flight magnetic resonance angiography (TOF-MRA; Figure 2). Here a presaturation pulse is applied directly to the bulk water signal in the image slice of interest, thereby eliminating the water signal. A small time delay then allows any mobile water protons, such as those in blood, to move out of the slice and be replaced by new blood containing spins that were not saturated. An image is then acquired in which the signal from the stationary tissue is darkened by saturation, whereas the signal of blood appears at normal intensity. The result is dramatic, showing a nice delineation of the vascular space from the surrounding tissue (Figure 2). More recently, $\mathrm{Gd}^{3+}$-based $\mathrm{T}_{1}$ agents that enhance the blood signal by binding to albumin have been introduced for contrast enhanced MR angiography (CE-MRA), providing an additional option for imaging the vasculature $(27,28)$.

One recent challenge has been the development of new imaging agents for cell tracking. Exogenous paramagnetic agents of various types have been loaded into cells at levels sufficient to alter $T_{1}$ or $T_{2}$ without overt loss of biological function (29-32). However, these contrast effects tend to fade with time either because of cell leakage or cell division. van Zijl and coworkers reported an ingenious approach to circumvent these problems by genetically altering glioma cells to express a lysine-rich protein (LRP) that can then be detected by CEST imaging (33). The large number of equivalent amide protons in the LRP appearing $3.76 \mathrm{ppm}$ downfield of the water resonance were used to initiate CEST (34). Genetically modified giloma cells, 
implanted into a mouse brain, were easily differentiated from control cells by CEST imaging by applying a selective presaturation pulse at the resonant frequency of the LRP amide resonance (Figure 3).

The images show CEST contrast only in the region where the engineered cells had been implanted, demonstrating that unique CEST activation systems such as this may be useful for cell tracking. Because the LRP is produced by the genetic machinery of the cell, its concentration should, in principle, be maintained even after many cell divisions. Furthermore, different cells could be encoded to produce different markers for CEST that require different activation frequencies. This would allow two, or more, types of cells to be tracked simultaneously by applying an appropriate frequency-selective presaturation pulse to follow the cells of interest.

This example demonstrates one of the main advantages of CEST imaging-CEST contrast can be turned on selectively by simply adding a presaturation pulse to an imaging sequence. The apparent negative image contrast generated by CEST activation is not a drawback in this case because a control CEST image that uses a different activation frequency (typically at an equal frequency offset on the opposite side of the water frequency) must be gathered so the difference image can be displayed as a positive image, a negative image, or a colorized image.

In mammalian tissue, excess glucose is stored in the form of the branching polymer glycogen (Figure 4). The body uses glycogen to replenish blood glucose levels as necessary, so a noninvasive method of tracking the distribution, synthesis, and breakdown of glycogen could have a major impact as a diagnostic tool in humans with glycogen storage disease or diabetes. The size and degree of branching of glycogen can vary but typically it has a molecular weight of several million and, by definition, a large number of exchangeable hydroxyl protons. Presaturation of glycogen -OH resonances $\sim 1 \mathrm{ppm}$ downfield of water enables these hydroxyl protons to be used as CEST antennae (35). The CEST spectra of glycogen recorded at $4.7 \mathrm{~T}$ and 9.4 $\mathrm{T}$ shown in Figure 4 nicely illustrate the advantage of working at higher magnetic fields for CEST detection. The value of $\Delta \omega$ (measured in Hertz) is larger at the higher field, and this renders the slow-to-intermediate exchange condition more favorable as well.

CEST arising from glycogen (glycoCEST) can be used to follow glycogen breakdown (glycogenolysis) stimulated by the hormone glucagon. The liver of a fed mouse has a substantial amount of glycogen that can easily be degraded and exported for use by other tissues. During periods of starvation, blood glucose levels begin to drop and the pancreas responds by releasing glucagon. Glucagon, in turn, binds to specific receptors on the surface of liver cells, and through a cascade of intracellular events, this stimulates glycogenolysis and export of glucose. These same events occur when glucagon is presented to an isolated perfused liver, but, in this case, glucose is simply exported to the surrounding perfusate. A series of colorized glycoCEST images of liver from a fed mouse are shown (Figure 4, bottom). The color variations in these images suggest that glycogen may not be uniformly distributed throughout the liver, or that proton exchange rates may vary depending on the location or size of the glycogen particle. Differentiating between these possibilities will require further detailed studies of the CEST properties of various chemical forms of glycogen. Nevertheless, glycoCEST images acquired at time intervals after administration of glucagon demonstrate that the magnitude of CEST is reduced as glycogen is broken down and glucose is released from the liver.

Although glycogen can be detected by CEST activation of a large population of hydroxyl protons, the protons of many alcohols and amines are not generally amenable to CEST because chemical exchange from these types of functional groups is often too fast $(34,36)$. Amide protons, however, exchange more slowly and represent an attractive means of generating CEST 
contrast (37). We have already seen the effect of irradiating the amide protons of LRP in cells modified to overexpress that protein. However, naturally occurring amide protons are abundant in all tissue and can be used to obtain information about the state of the tissue through CEST imaging. The rate of amide proton exchange is highly dependent on $\mathrm{pH}$; above approximately $\mathrm{pH} 5$, the presence of base catalyses exchange, so the higher the $\mathrm{pH}$, the faster the exchange rate. This difference in exchange rate can be used to assess $\mathrm{pH}$ because the magnitude of CEST is strongly influenced by the exchange rate. Ischemic regions of rat brain tissues have been imaged by applying a presaturation pulse at 3.5 (sat-on) and $-3.5 \mathrm{ppm}$ (sat-off) to probe the CEST arising from naturally abundant amide protons (38). Regions of ischemia were clearly distinguished in the CEST images, presumably reflecting $\mathrm{pH}$ differences due to a loss of tissue oxygenation and aerobic metabolism. No such tissue differentiation was observed in $\mathrm{T}_{2^{-}}$ weighted control images, demonstrating that CEST imaging could provide additional diagnostic utility over conventional imaging methods.

\section{EXOGENOUS CHEMICAL EXCHANGE SATURATION TRANSFER AGENTS}

The CEST applications discussed so far have focused on the use of endogenous species having a component in exchange with the solvent water. However, there are circumstances when one may wish to obtain information that cannot be gained from an endogenous CEST probe. Here, it may be desirable to administer an exogenous CEST agent that can respond to some biological event or change in physiology. We describe later how exogenous agents can be used to effect changes in the water signal intensity, but we first consider a recently reported example of CEST that uses an NMR nucleus not naturally found in tissue (39). ${ }^{129} \mathrm{Xe}$ has a favorable NMR spin quantum number of $1 / 2$, its chemical shift is extremely sensitive to its chemical environment, and it has an unusually long $\mathrm{T}_{1}$. Xenon gas can be inhaled safely and because there is zero background signal in tissue, it has been considered for use in lung imaging. Xenon is soluble in both water and fat, so this inert substance distributes to some extent in all tissues (40).

Because the chemical shift of ${ }^{129} \mathrm{Xe}$ is exquisitely sensitive, gaseous xenon, xenon dissolved in blood, and xenon in various tissue compartments are easily discriminated.

Unfortunately, ${ }^{129} \mathrm{Xe}$ is not the most sensitive of NMR nuclei (similar sensitivity as ${ }^{13} \mathrm{C}$ ), so the amount that can be delivered as a gas barely provides enough signal for imaging. However, the sensitivity of this nucleus can be improved dramatically by first hyperpolarizing the ${ }^{129} \mathrm{Xe}$, typically by optical pumping of rubidium gas followed by polarization transfer (spin exchange) to ${ }^{129} \mathrm{Xe}(40,41)$. The effect of hyperpolarization is the reverse of saturation; the number of spins aligned with the magnetic field is increased, relative to the normal Boltzmann distribution, and thus the net magnetization is also considerably enhanced. The sensitivity of ${ }^{129} \mathrm{Xe}$ can be increased up to 100,000 -fold making it possible to image inhaled xenon gas with exquisite image resolution. Of course, the magnetization of a hyperpolarized nucleus returns to steady-state Boltzmann levels with a rate constant of $1 / T_{1}$, so the amount of time one has available for imaging heavily depends on the $T_{1}$ relaxation rate of the nucleus.

Because ${ }^{129} \mathrm{Xe}$ has a long $\mathrm{T}_{1}$ over one minute in water at $9.4 \mathrm{~T}$, there is considerable interest in the possibility of pulmonary imaging with hyperpolarized ${ }^{129} \mathrm{Xe}$.

$\mathrm{Xe}$ is considered to be chemically inert because it is not easily oxidized or reduced and only forms stable covalent bonds with certain elements. However, Xe can be encapsulated by chemical cages of appropriate size via van der Waals interactions, and such caged complexes can, in principle, be covalently linked to a targeting motif for delivery of hyperpolarized ${ }^{129} \mathrm{Xe}$ to a specific tissue target. Encapsulated Xe experiences a sizable shift in resonance frequency of more than $100 \mathrm{ppm}$, so it is easy to discriminate between encapsulated and free Xe. Although these cages may be able to localize Xe to a specific tissue type, even with the benefits of hyperpolarization many biologically relevant targets, such as cell receptors, are present in such low concentrations (often nanomolar or lower) that direct detection of the target-bound encapsulated Xe would not be possible. However, the rate of 
exchange between free and encapsulated forms of Xe was found to be slow on the NMR timescale, and this raised the possibility of performing CEST experiments with hyperpolarized $\mathrm{Xe}$ to detect the minor species (caged $\mathrm{Xe}$ ) through the signal of the more abundant species (free Xe) (39).

Figure 5 shows images of a phantom system of agarose beads, one half of which are plain agarose beads and the other half of which are avadin-agarose beads. The beads were treated with crytophanes conjugated, through a water-soluble linker, to biotin, which selectively binds with avidin. The system was then perfused with hyperpolarized Xe-saturated water and images collected. When the phantom was imaged after presaturation of the encapsulated Xe (65.4 $\mathrm{ppm}$ ), a significant decrease in the signal of free Xe (193.6 ppm) was observed (Figure 5). This system nicely demonstrates that this hyperCEST technique is able to differentiate the more abundant free Xe at sensitivity levels characteristic of hyperpolarized nuclei even though the caged atoms are barely detectable by direct ${ }^{129} \mathrm{Xe}$ NMR spectroscopy. Furthermore, xenon biosensors such as this could be preloaded to allow time for tissue targeting prior to diffusion of hyperpolarized Xe.

\section{Paramagnetic Chemical Exchange Saturation Transfer Agents}

The development of hyperpolarization technologies and hyperCEST present new and exciting opportunities for MRI using a wide range of NMR active nuclei. However, clinical MR imaging largely involves detection of protons, so it is important to remain focused on CEST agents that can provide useful clinical information by altering contrast in water proton images. Balaban and coworkers were first to propose using low-molecular-weight, diamagnetic molecules with exchangeable protons as exogenous CEST agents $(42,43)$. A number of sugars, metabolites, amino acids, and other small molecules were evaluated by recording a CEST spectrum in which the intensity of the bulk water signal is plotted as a function of the presaturation frequency. Many of these small diamagnetic molecules showed a significant ability to alter the solvent water signal intensity at the relatively high concentrations of CEST agent $(10-100 \mathrm{mM})$. The CEST spectrum of barbituric acid, a typical diamagnetic agent, is shown in Figure 6. From this spectrum, it is clear that the shift difference between the two exchanging pools is small, $\sim 5$ ppm. This limits the CEST experiment in two ways: $(a)$ application of a presaturation pulse even $5 \mathrm{ppm}$ away from the bulk water signal will result in some off-resonance saturation of the water so there are limitations in the amount of power that can be applied, and $(b)$ the slow exchange condition $\left(\mathrm{k}_{\mathrm{ex}}<\Delta \omega\right)$ means that very slow exchange rates are required for diamagnetic CEST agents of this sort. Both problems can be alleviated by using an agent that has a larger chemical shift separation between the two exchanging pools, much like the Xe example described above. This allows a broader spectrum of suitable exchange agents to be investigated and, intuitively, more rapid chemical exchange will increase the number of saturated spins that can be transferred to the solvent water in any given time frame, thereby increasing the observed CEST effect.

The rate of water exchange in the $\mathrm{Gd}^{3+}$ complexes used as $\mathrm{T}_{1}$-shortening MRI contrast agents is quite fast $\left(1 / \mathrm{k}_{\mathrm{ex}} \ll 1 \mu \mathrm{s}\right)$ and certainly too fast for CEST $(1,44)$. However, the structure of these complexes can be modified in such a way that favors much slower exchange kinetics while retaining the stability of the complex required for in vivo applications (45-47). The discovery of such slow water exchange complexes suggested to us that such lanthanide-based chelates might be used as paramagnetic CEST agents (PARACEST) $(48,49)$. The $\mathrm{Gd}^{3+}$ ion is isotropic (seven unpaired electrons in seven $4 \mathrm{f}$ orbitals) so it cannot act as a shift reagent, but other paramagnetic lanthanide ions with anisotropic distributions of their f-electrons can induce very large shifts in the resonance frequencies of proximate protons (50-52). Some of those other lanthanides, when complexed with DOTA-tetraamide ligands, exhibit a single, highly shifted metal ion-bound water resonance in their ${ }^{1} \mathrm{H}$ NMR spectra due to slow water exchange 
(49). The chemical shift of this metal ion-bound water molecule can vary from +500 to -720 ppm depending on which lanthanide ion is coordinated (53). The first paramagnetic complex that showed a water-exchange peak in its CEST spectrum was in EuDOTA-(glycine ethyl ester) ${ }_{4}$ (Figure 6) (48). Here, an exchange peak near $+50 \mathrm{ppm}$ was easily assigned to a water molecule that exchanges between an inner-sphere coordination position on the $\mathrm{Eu}^{3+}$ and bulk solvent. The exchange peak observed in this spectrum was consistent with models presented earlier, suggesting that water-exchange rates would vary with the bulkiness of the appended amide side chain groups (45). Highly shifted exchange peaks such as this offer a distinct advantage for CEST activation in that direct off-resonance saturation of the bulk water pool is largely eliminated, in dramatic contrast to diamagnetic CEST agents (Figure 6). Such large $\Delta \omega$ values also expand the range of $k_{\mathrm{ex}}$ values allowable for CEST. Although the hyperfine shifted exchange resonances seen in $\mathrm{Eu}^{3+}$ complexes are smaller than those found in the analogous $\mathrm{Tm}^{3+}(+500 \mathrm{ppm})$ and $\mathrm{Dy}^{3+}(-720 \mathrm{ppm})$ complexes, $\mathrm{Eu}^{3+}$ complexes have been more widely studied as PARACEST agents. The reasons for this popularity relate to the fact that water exchange is found to be slowest in $\mathrm{Eu}^{3+}$ complexes in comparison to all other lanthanides (54) and to the poor relaxation properties of $\mathrm{Eu}^{3+}$ that result in long relaxation times, which in turn favor CEST

As other ligands and metal ions have been studied, it has been found that CEST can be achieved by presaturating other exchangeable protons in lanthanide complexes, in particular, coordinating alcohol protons (55), coordinating amide protons (56-58), and proximate noncoordinating amide protons $(59,60)$. These discoveries have had important consequences for the development of PARACEST agents as an imaging tool. The primary advantage of CEST agents, and in particular PARACEST agents, over conventional $\mathrm{T}_{1}$-shortening agents is in the amount and type of information that can be obtained. In particular, metabolic information, which we have already seen is difficult to acquire with $\mathrm{Gd}^{3+}$-based contrast agents, is more easily obtained using a PARACEST agent. Because CEST is extremely sensitive to exchange rates, any change in the prevailing conditions that alters the exchange rate is likely to have a profound effect on CEST. We first examine the practical advantages of these agents before discussing some design features that provide the basis for these responsive agents.

Two experimental conditions are intrinsically linked to the rate of proton and water exchange, sample temperature and $\mathrm{pH}$. The exchange rate of all species increases with increasing temperature according to Arrhenius's law, so PARACEST agents such as these have potential as MRI temperature probes $(57,61)$. In contrast, the relationship between exchange rate and $\mathrm{pH}$ can be more complex (57). For example, the rate of metal-bound water proton exchange is unaffected by changes in $\mathrm{pH}$ between 2 and 8 but increases at higher $\mathrm{pH}$, whereas the rate of coordinated amide proton exchange increases significantly between $\mathrm{pH} 5$ and 8 . Aime and coworkers have proposed methods by which the $\mathrm{pH}$ dependence of CEST from the amide protons in lanthanide DOTA-tetraamide complexes might be used to assess $\mathrm{pH}$ by MRI in a concentration-independent manner (58). The CEST effect arising from metal-bound water exchange in EuDOTA-tetraamide complexes $(+50 \mathrm{ppm})$ is unaffected by changes in $\mathrm{pH}$ over the physiological $\mathrm{pH}$ range so it has been proposed as a concentration marker. In contrast, the CEST effect from the coordinating amide protons of the $\mathrm{Yb}^{3+}$ complex of the same ligand $(-20$ $\mathrm{ppm}$ ) will report changes in $\mathrm{pH}$. $\mathrm{Eu}^{3+}$ and $\mathrm{Yb}^{3+}$ complexes are isostructural so one might anticipate they would distribute in tissue identically. Hence, a cocktail of $\mathrm{Eu}^{3+}$ and $\mathrm{Yb}^{3+}$ complexes with the same ligand could be administered simultaneously and CEST activation at $+50 \mathrm{ppm}$ would report the agent concentration without activating the $\mathrm{pH}$ reporter, whereas CEST activation at $-20 \mathrm{ppm}$ would report the $\mathrm{pH}$ (Figure $7 a$ ). The large difference in irradiation frequency between the amide and metal-bound water protons allows each pool to be activated selectively without influencing the other exchange site. CEST from both the amide and water protons can be observed for many $\mathrm{Eu}^{3+}$ DOTA-tetraamide complexes so it may not be necessary to use two lanthanide ions complexes to achieve this goal. However, the intensity 
of each CEST peak, water and amide, must be comparable if one is to be used as a concentration marker. For $\mathrm{Eu}^{3+}$ complexes this condition is not met, but by using a mixture of two complexes, the composition of the cocktail can be tuned to achieve the desired balance. Aime and coworkers have subsequently reported that a single complex can provide the desired balance in CEST intensities between the two presaturation sites (57). They showed that a DOTAtetraamide complex of $\mathrm{Pr}^{3+}$ is able to provide a $\mathrm{pH}$ sensing function through its amide protons and a concentration determination through its coordinated water molecule. Although these two site probes for metabolic information are quite viable in an imaging context, a simpler, more elegant solution that requires only a single exchanging pool has been proposed by Wegh and coworkers (62). In their work, changes in $\mathrm{pH}$ were assessed using a dendrimer-like compound with several YbDOTA-tetraamide complexes appended, the amide protons of which have $\mathrm{pH}$ sensitive CEST properties (63). However, their approach to measuring changes in $\mathrm{pH}$ can, in principle, be extended to any responsive CEST agent. As an example, Figure 7 shows the CEST spectra of a EuDOTA-tetraamide complex recorded at different temperatures (61). It can be seen that the CEST peak corresponding to the metal-bound water decreases in intensity as the temperature increases, but, as it does so, it also broadens and shifts toward the bulk water peak. This NMR signature is characteristic of a system undergoing more rapid chemical exchange with increasing temperature. If the magnitude of the CEST effect is measured by presaturation at $+51 \mathrm{ppm}$, it will decrease with increasing temperature. However, a significant CEST effect can also be generated by presaturating closer to the solvent water frequency, say $+38 \mathrm{ppm}$, and the response of the CEST magnitude after presaturation at this frequency is completely different from that at $+51 \mathrm{ppm}$. So by taking the ratio of the change in solvent water signal intensity after presaturation at $+51 \mathrm{ppm}$ and $+38 \mathrm{ppm}$, both acting on the metal-bound water, a concentration-independent method of determining temperature can be obtained (Figure $7 c$ ). The benefit of this method is that only one exchanging pool that responds to the parameter of interest is required.

Clearly, the insensitivity of the metal-bound water-exchange peak in EuDOTA-tetraamide complexes to changes in $\mathrm{pH}$ makes this an ideal platform to design agents that respond to other physiological or biological parameters. To design such an agent, one must first choose a complex in which the exchange rate of the metal-bound water is affected by its interaction with the species under consideration. In principle, there are three routes to achieving this goal: $(a)$ alter the steric encumbrance around the water molecule, $(b)$ introduce a group that catalyzes proton or water exchange, or $(c)$ alter the interaction binding energy between the metal ion and the water molecule. Let's consider each in turn. A PARACEST agent may be designed in such a way that the species under analysis binds, either covalently or noncovalently, to the complex above the coordination site of metal-bound water. In doing so, it makes it harder for the metalbound water molecule to exchange with the bulk simply because the analyte is impeding departure of the water molecule from its metal-binding site. This should result in a decreased water-exchange rate in response to exposure to the analyte of interest. One example of such as system is a recently reported glucose-responsive PARACEST agent $(64,65)$ in which the glucose, binding to two phenyl boronates, sits above the water coordination site and slows water exchange. In this example, water exchange slows by approximately a factor of two when glucose is bound and this is sufficient to switch on CEST from the metal-bound water. Figure $8 a$ shows images of the binding event in phantom systems but monitoring glucose distribution in isolated, perfused mouse livers has also been performed (J. Ren \& A.D. Sherry, unpublished data).

Certain proximate groups, such as phosphonates, can catalyze the exchange of metal-bound water protons, a feature that has been used to design $\mathrm{pH}$ responsive $\mathrm{Gd}^{3+}$ agents (3). If, upon binding, the chosen analyte introduces a suitably located group that is capable of catalyzing water proton exchange, then the resulting increase in apparent water-exchange rate will be reflected in the CEST properties of the PARACEST agent. A PARACEST agent with four 
pyridyl groups was designed to bind the $\mathrm{Zn}^{2+}$ ion above the water coordination site (66), but unlike the glucose sensor described above, at physiological $\mathrm{pH}$ the $\mathrm{Zn}^{2+}$ ion bound to this complex presents a coordinated hydroxyl group just above the $\mathrm{Eu}^{3+}$-bound water molecule, which catalyzes acceleration of water proton exchange, thereby affording very large changes in the CEST spectrum upon binding of $\mathrm{Zn}^{2+}$. In this case, the CEST was on initially in the absence of $\mathrm{Zn}^{2+}$ but was turned off when the catalytic-OH group was introduced by the $\mathrm{Zn}^{2+}$.

Water exchange in EuDOTA-tetraamide complexes such as these is known to occur by a dissociative mechanism, whereby the single bound water molecule must leave the $\mathrm{Eu}^{3+}$ coordination sphere before a different water molecule can enter (67). Consequently, the strength of the interaction between the $\mathrm{Eu}^{3+}$ and its bound water molecule essentially determines the water-exchange rate. If the strength of this interaction can be subtly altered in response to changes in concentration of a parameter of interest, then this might also be reflected in the water-exchange rate and, ultimately, in the CEST properties of the complex. To test this hypothesis, a series of complexes were prepared in which one amide substituent was systematically altered to vary the electron-donating power of that amide ligand (68). The magnitude of the CEST effect was found to vary with the nature of the amide substituent, supporting the hypothesis that these electronic effects can be used to design responsive PARACEST agents. As shown in Figure $8 c$, when the amide substituent was varied from a $p$ $\mathrm{NO}_{2}$-phenyl (electron-withdrawing) to a $p-\mathrm{NH}_{2}$-phenyl (electron-donating) substituent, a significant difference in CEST image intensity is observed in phantom systems after presaturation of the metal-bound water molecule. This suggests that a generalized approach to the design of responsive agents may be possible that would even allow for assessment of parameters, such as tissue redox, for which it may otherwise have been difficult to design a responsive agent.

The design of responsive PARACEST agents is not restricted to examining changes in the signal arising from the metal-bound water protons or the coordinated amide protons. Pagel and coworkers adopted a somewhat different approach in developing a PARACEST agent that responds to the action of an enzyme (60). Rather than begin with a DOTA-tetraamide complex, as in the previous examples, the authors started with DOTA modified by a primary amine in the $\alpha$-position of one pendant arm. This amine was then used to attach a peptide chain that could be cleaved by caspase- 3 . The amide NH in the $\alpha$-position of DOTA could then be used as a CEST antennae in the same way we have seen amide protons used to generate CEST; however, in this case, its effectiveness was increased by incorporating a lanthanide with good shifting properties, $\mathrm{Tm}^{3+}$, to shift the -NH resonance as far away from water as possible, thereby magnifying $\Delta \omega$. A sizable CEST effect was observed for a $25 \mathrm{mM}$ solution of the complex arising from this single amide proton. However, after one hour in the presence of $48 \mathrm{nM}$ caspase-3, most of the peptide had been cleaved, leaving behind a primary amino group that showed no CEST because proton exchange was too fast in this case. The advantage of this approach is that the agent does not require stoichiometric binding of a substrate but rather the CEST magnitude depends only on the amount of time the agent is exposed to the enzyme and the enzyme turnover number or activity. The disadvantage of course is that such systems are not reversible. Nevertheless, the idea of creating enzyme-specific CEST agents that are activated by enzymatic cleavage of a specific bond is a good one and should provide added value to the arsenal of biological information provided by MRI.

One further development in the field of PARACEST agents worthy of mention at this point is targeted CEST agents. $\mathrm{Eu}^{3+}$ and $\mathrm{Yb}^{3+}$ complexes of DOTA-tetraamide ligands having one or more benzyloxy ether substituents for binding to serum albumin (69-71) have been reported (72). A number of $\mathrm{Gd}^{3+}$-based agents that bind to serum albumin have been observed to exhibit a decrease in the water-exchange rate upon binding $(69,70,73,74)$, and a similar effect was 
anticipated in the case of CEST agents. Quite unexpectedly, the rate of water exchange in these PARACEST agents was found to increase upon binding to serum albumin, likely due to catalysis of proton exchange by protein surface residues. This agent showed a detectable CEST effect at concentrations as low as $0.75 \mathrm{mM}$ when bound to albumin. This may be too high to allow these complexes to function as fully fledged blood-pool agents, but there may be some potential advantages to using CEST agents in other targeted contrast media applications. Conventional $\mathrm{T}_{1}$ or $\mathrm{T}_{2}$ MR contrast agents require image collection pre- and postcontrast so any motion artifacts can contribute to registration errors. CEST contrast agents offer the opportunity to interleave images by acquiring each projection with and without presaturation. The two images can effectively be acquired at the same time, reducing the effects of motion on any resulting difference image. This is the great advantage of being able to turn a contrast medium on/off at will by turning on/off the presaturation pulse.

\section{Sensitivity-Enhanced Chemical Exchange Saturation Transfer Agents}

One of the current limitations of exogenous CEST agents is their relatively low sensitivity, typically requiring $1-10 \mathrm{mM}$ of agent for detection. In an attempt to generate systems that are capable of operating at much lower detection limits and potentially image biologically relevant targets, several approaches to enhance the sensitivity of CEST agents have been proposed. Unlike $\mathrm{Gd}^{3+}$-based contrast agents, which require complicated design features to improve relaxivity, CEST agents can be made more sensitive by simply increasing the number of exchangeable groups on the agent. Lanza, Wickline, and coworkers used perfluorocarbonfilled nanoparticles, modified with a targeting ligand, to deliver large payloads of $\mathrm{Gd}^{3+}$ to specific sites (75-77). Recently, this work was extended by using an appropriately functionalized $\mathrm{Eu}^{3+}$ complex (78) to load the particles with CEST agents (79). Upward of $10^{5}$ chelates could be loaded onto the surface of each nanoparticle (77), thereby dramatically increasing the number of water-exchanging sites. This model PARACEST-loaded nanoparticle has been used to demonstrate that fibrin is easily detected in blood clots by CEST imaging (79).

Another strategy that has been used to increase the sensitivity of CEST agents is to encapsulate a small volume of highly shifted water within the core of a semipermeable membrane. Liposomes are ideal for this purpose because water exchange between the intra- versus extraliposomal compartments can be adjusted by varying the membrane composition. The first such example was a liposome in which the encapsulated water was shifted using the shift reagent, TmDOTMA (80). $\mathrm{Tm}^{3+}$ induced a significant downfield shift of the intraliposomal water, which could then be used as a CEST antenna. With an average liposome internal volume of $9.2 \times 10^{-18}$ liters, the number of exchanging sites available for CEST is increased from 2 (for a single water molecule) to approximately $1.1 \times 10^{10}$ in the liposome (Figure $9 b$ ). This lowered the detection limit for a LIPOCEST agent of this type to $\sim 90 \mathrm{pm}$. This concept of using liposomes as CEST agents was extended recently by encapsulating the clinically approved contrast agent, ProHance (GdHP-DO3A), into liposomes (81). As indicated previously, the isotropic $\mathrm{Gd}^{3+}$ ion does not induce paramagnetic shifts, so no shift is observed for spherical liposomes containing the agent. However, the liposome shape can be altered through changes in osmolality; adding sodium chloride to the external water causes the liposome to expel water from its inner core, shrinking and deforming the liposomes into nonspherical shapes. By increasing the osmolality of the external solution in this way, the liposomes themselves become anisotropic and this results in a shift in the intraliposomal water resonance induced by the isotropic $\mathrm{Gd}^{3+}$ agent (Figure $9 c$ ). Such liposomes can be considered a dual modality MR contrast agent because they act both as an effective CEST agent and as a $\mathrm{T}_{1}$-shortening agent. At isotonic concentrations of sodium chloride ( $\left.300 \mathrm{mOsmol}\right)$, the liposomes were estimated to have a detection limit of $\sim 30 \mathrm{nM}$ by CEST imaging, a reasonable range to facilitate imaging of some cell receptors. 
An alternative to increasing the number of small molecules with exchanging sites, e.g., water or metal-bound water, is to use a system that inherently has an extremely large number of exchangeable protons. Clearly this requires the use of macromolecular structures, although in certain applications, it is these very macromolecules that are of interest. One such example is the field of gene therapy, where the in vivo fate of therapeutic nucleic acids is of considerable interest. Nucleic acids are anionic polymers, and thus a delivery system is typically required to transport the nucleic acid polymer across the cell membrane and into the cell nucleus. Often viruses are used for this purpose, but nucleic acids may also be delivered by bundling them with cationic polymers. These cationic polymers may contain several kinds of exchanging protons; most contain a protonated amine, which exchanges protons too rapidly for CEST $(34,36)$, but a number of polymers used for gene therapy also contain amides. Polycations, such as poly-L-lysine (PLL) and starburst PAMAM dendrimers, contain amide groups and both examples have been considered for use in gene delivery $(34,36)$. A representative CEST spectrum of PLL is shown in Figure 10. The large numbers of amide groups present in these structures means that even at concentrations of $0.1 \mathrm{mM}$, the solvent water signal can be reduced in intensity by almost half. This opens the possibility of using a cationic delivery system as a method of tracking nucleic acids during gene therapy. However, a more direct approach to tracking gene delivery that would not limit the choice of delivery platform may be available. Snoussi et al. subsequently examined poly(rU)to see if any of the exchangeable groups present in the nucleic acid strand could be used to activate CEST contrast (26). Two protons in a strand of poly(rU) were found to give rise to CEST, the hydroxyl group of the ribose moiety and the $\mathrm{NH}$ of the uracil base. The CEST effects arising from these two protons are clearly identifiable in the CEST spectrum (Figure 10). Like the cationic polymers, large numbers of these protons are present in the nucleic acids used for gene therapy, so the detection limit for CEST agents of this type is likely to be quite low. Interestingly, the CEST effects from poly(rU) could be detected even with the nucleic acid bundled with a PAMAM dendrimer (26). Using the exchangeable pools of the nucleic acid as antennae, the dendrimer-poly(rU) bundle has proven even more sensitive than PLL, providing a comparable change in water signal intensity at just $10 \mu \mathrm{M}$.

\section{CONCLUDING REMARKS}

Although $\mathrm{Gd}^{3+}$-based $\mathrm{T}_{1}$ agents are widely accepted both in basic research and in clinical medicine, the era of molecular medicine will require new information that is not typically provided by anatomical imaging. A new class of paramagnetic lanthanide complexes having slowly exchanging water molecules or other types of $-\mathrm{NH}$ or $-\mathrm{OH}$ protons provides a new avenue to introduce tissue contrast via a CEST mechanism. Because MR contrast provided by PARACEST agents is determined by chemical features, such as water molecule exchange or proton exchange, rather than by physical characteristics of the paramagnetic center, they offer new opportunities for creating responsive CEST agents capable of reporting significant biological or physiology events by MRI. Early studies have shown that exchange in these systems is easily modulated by simple chemical manipulation, so applying fundamental chemical principles, such as molecular recognition or self-assembly, to these systems should prove invaluable in creating novel systems for reporting molecular events by MRI.

\section{SUMMARY POINTS}

1. A new class of MRI contrast agents based on a chemical exchange saturation transfer (CEST) mechanism offers new opportunities for development of MR gene reporters and biologically responsive imaging agents.

2. CEST contrast can arise from multiple types of proton exchange groups, so molecules endogenous to tissues as well as exogenous agents are potential imaging candidates. 
3. A large chemical shift difference between an exchanging proton site and solvent water $(\Delta \omega)$ is highly advantageous for CEST contrast because the exchange site can be activated using a selective RF pulse without indirectly partially saturating water.

4. Paramagnetic lanthanide ions that induce large hyperfine shifted resonances in nearby protons are especially useful for CEST contrast because large $\Delta \omega$ values allow access to a wider variety of faster exchanging chemical systems. Lanthanidebased agents of this type are referred to as PARACEST agents.

5. Numerous biological and chemical approaches are being taken to enhance the sensitivity of both endogenous and exogenous CEST agents.

\section{Glossary}

Responsive agent, a contrast agent that affects image contrast differently depending on the presence of another biological species or a change in some physiological or biochemical parameter, such as $\mathrm{pH}, \mathrm{O}_{2}$ tension, or enzyme activity

Chemical exchange saturation transfer (CEST), causes a reduction in the signal intensity of an NMR resonance that is in chemical exchange with another resonance to which a low-power irradiation or presaturation pulse has been applied

Saturation, a condition wherein the number of nuclear spins aligned against the field is increased at the expense of those aligned with the field, leading to a decrease in signal intensity Boltzmann distribution, the ratio of the spins aligned with the field over those aligned against= $\mathrm{e}^{(-\Delta \mathrm{E} / \mathrm{kT})}$

$\Delta \omega$, the difference between the resonance frequencies of two pools involved in chemical exchange

LRP, lysine-rich protein

Hyperpolarization, a condition wherein the number of nuclear spins aligned with the field is increased at the expense of those aligned against the field, leading to an increase in signal intensity

Paramagnetic CEST (PARACEST), chemical exchange saturation transfer that arises from presaturation of exchangeable protons on a paramagnetic molecule or complex

DOTA-tetraamide, an octadentate ligand based on the structure 1,4,7,10-

tetraazacyclododecane-1,4,7,10-tetraacetamide

Arrhenius's law, $k_{e x}=A e^{\left(-E_{a} / R T\right)}$

Isostructural, having identical structures despite differences in chemical composition

\section{ACKNOWLEDGMENTS}

This review was supported in part by grants from the National Institutes of Health (CA-115531, DK-058398 and RR-02584) and the Robert A. Welch Foundation (AT-584).

\section{LITERATURE CITED}

1. Caravan P, Ellison JJ, McMurry TJ, Lauffer RB. Gadolinium(III) chelates as MRI contrast agents: structure, dynamics, and applications. Chem. Rev 1999;99:2293-1352. [PubMed: 11749483]

2. Toth, E.; Merbach, AE. Chemistry of Contrast Agents in Medical Magnetic Resonance Imaging. New York: Wiley; 2001.

3. Kalman FK, Woods M, Caravan P, Jurek P, Spiller M, et al. Potentiometric and relaxometric properties of a gadolinium-based MRI contrast agent for sensing tissue pH. Inorg. Chem 2007;46:5260-5270.

[PubMed: 17539632] 
4. Aime S, Barge A, Botta M, Howard JAK, Kataky R, et al. Dependence of the relaxivity and luminescence of gadolinium and europium amino-acid complexes on hydrogencarbonate and $\mathrm{pH}$. Chem. Commun 1999;1999:1047-1048.

5. Lowe MP, Parker D. Controllable $\mathrm{pH}$ modulation of lanthanide luminescence by intramolecular switching of the hydration state. Chem. Commun 2000;2000:707-708.

6. Aime S, Crich SG, Botta M, Giovenzana G, Palmisano G, Sisti M. A macromolecular Gd(III) complex as pH-responsive relaxometric probe for MRI applications. Chem. Commun 1999;1999:1577-1578.

7. Hovland R, Glogard C, Aasen AJ, Klaveness J. Gadolinium DO3A derivatives mimicking phospholipids; preparation and in vitro evaluation as $\mathrm{pH}$ responsive MRI contrast agents. J. Chem. Soc., Perkin Trans 2001;2:929-933.

8. Glogard C, Hovland R, Fossheim SL, Aasen AJ, Klaveness J. Synthesis and physicochemical characterisation of new amphiphilic gadolinium DO3A complexes as contrast agents for MRI. J. Chem. Soc., Perkin Trans 2000;25:1047-1052.

9. Woods M, Kiefer GE, Bott S, Castillo-Muzquiz A, Eshelbrenner C, et al. Synthesis, relaxometric and photophysical properties of a new $\mathrm{pH}$-responsive MRI contrast agent: the effect of other ligating groups on dissociation of a p-nitrophenolic pendant arm. J. Am. Chem. Soc 2004;126:9248-9256. [PubMed: 15281814]

10. Hanaoka K, Kikuchi K, Urano Y, Nagano T. Selective sensing of zinc ions with a novel magnetic resonance imaging contrast agent. J. Chem. Soc., Perkin Trans 2001;2:1840-1843.

11. Li W-H, Fraser SE, Meade TJ. A calcium-sensitive magnetic resonance imaging contrast agent. J. Am. Chem. Soc 1999;121:1413-1414.

12. Li W-H, Parigi G, Fragai M, Luchinat C, Meade TJ. Mechanistic studies of a calcium-dependent MRI contrast agent. Inorg. Chem 2002;41:4018-4024. [PubMed: 12132928]

13. Dickins RS, Gunnlaugsson T, Parker D, Peacock RD. Reversible anion binding in aqueous solution at a cationic heptacoordinate lanthanide center: selective bicarbonate sensing by time-delayed luminescence. Chem. Commun 1998;1998:1643-1644.

14. Dickins RS, Love CS, Puschmann H. Bidentate lactate binding in aqueous solution in a cationic, heptadentate lanthanide complex: an effective chiral derivatising agent. Chem. Commun 2001;2001:2308-239.

15. Moats RA, Fraser SE, Meade TJ. A "smart" magnetic resonance imaging agent that reports on specific enzymatic activity. Angew. Chem. Int. Ed 1997;36:726-7228.7228 A seminal paper that first brought the idea of responsive contrast agents to light.

16. Anelli PL, Bertini I, Fragai M, Lattuada L, Luchinat C, Parigi G. Sulfonamide-functionalized gadolinium DTPA complexes as possible contrast agents for MRI: a relaxometric investigation. Eur. J. Inorg. Chem 2000;4:625-630.

17. Nivorozhkin AL, Kolodziej AF, Caravan P, Greenfield MT, Lauffer RB, McMurry TJ. Enzymeactivated $\mathrm{Gd}^{3+}$ magnetic resonance imaging contrast agents with a prominent receptor-induced magnetization enhancement. Angew. Chem. Int. Ed 2001;40:2903-2906.

18. De Leon-Rodriguez LM, Ortiz A, Weiner AL, Zhang S, Kovacs Z, et al. Magnetic resonance imaging detects a specific peptide-protein binding event. J. Am. Chem. Soc 2002;124:3514-3515. [PubMed: 11929234]

19. Garcia-Martin ML, Martinez GV, Raghunand N, Sherry AD, Zhang S, Gillies RJ. High resolution pH imaging of rat glioma using pH-dependent relaxivity. Magn. Reson. Med 2006;55:309-315. [PubMed: 16402385]

20. Raghunand N, Howison C, Sherry AD, Zhang S, Gillies RJ. Renal and systemic pH imaging by contrast-enhanced MRI. Magn. Reson. Med 2003;49:249-257. [PubMed: 12541244]

21. McConnell HM, Thompson DD. Molecular transfer of nonequilibrium nuclear spin magnetization. J. Chem. Phys 1957;26:958-959.

22. McConnell HM, Thompson DD. Molecular transfer of nonequilibrium nuclear spin magnetization. J. Chem. Phys 1959;31:85-88.

23. Forsén S, Hoffman RA. Study of moderately rapid chemical exchange reactions by means of nuclear magnetic resonance double resonance. J. Chem. Phys 1963;39:2892-2901. 
24. Woods M, Woessner DE, Sherry AD. Paramagnetic lanthanide complexes as PARACEST agents for medical imaging. Chem. Soc. Rev 2006;35:500-511.511 [PubMed: 16729144] A useful review for a more basic, and also more thorough, discussion of the theory behind CEST.

25. Woessner DE, Zhang S, Merritt M, Sherry AD. Numerical solution of the Bloch equations provides insights into the optimum design of PARACEST agents for MRI. Magn. Reson. Med 2005;53:790799.799 [PubMed: 15799055] Presents a numerical solution of the Bloch equations used to fit CEST spectra.

26. Snoussi K, Bulte JWM, Gueron M, van Zij1 PCM. Sensitive CEST agents based on nucleic acid imino proton exchange: detection of poly $(\mathrm{rU})$ and of a dendrimer-poly $(\mathrm{rU})$ model for nucleic acid delivery and pharmacology. Magn. Reson. Med 2003;49:998-1005. [PubMed: 12768576]

27. Meaney JF. Magnetic resonance angiography of the peripheral arteries: current status. Eur. Radiol 2003;13:836-852. [PubMed: 12664125]

28. Eiberg JP, Lundorf E, Thomsen C, Schroeder TV. Peripheral vascular surgery and magnetic resonance arteriography—a review. Eur. J. Vasc. Endovasc. Surg 2001;22:396-402. [PubMed: 11735175]

29. Bhakoo, K.; Chapon, C.; Jackson, J.; Jones, W. Application of MRI to cell tracking. In: Webb, G., editor. Modern Magnetic Resonance. New York: Springer; 2006. p. 873-884.

30. Rogers WJ, Meyer CH, Kramer CM. Technology insight: in vivo cell tracking by use of MRI. Nat. Clin. Pract. Cardiovasc. Med 2006;3:554-562. [PubMed: 16990841]

31. Arbab AS, Liu W, Frank JA. Cellular magnetic resonance imaging: current status and future prospects. Expert Rev. Med. Devices 2006;3:427-439. [PubMed: 16866640]

32. Bulte JWM, Kraitchman DL. Monitoring cell therapy using iron oxide MR contrast agents. Curr. Pharm. Biotechnol 2004;5:567-584. [PubMed: 15579045]

33. Gilad AA, McMahon MT, Walczak P, Winnard PT Jr, Raman V, et al. Artificial reporter gene providing MRI contrast based on proton exchange. Nat. Biotech 2007;25:217-219.

34. Goffeney N, Bulte JWM, Duyn J, Bryant LH Jr, van Zijl PCM. Sensitive NMR detection of cationicpolymer-based gene delivery systems using saturation transfer via proton exchange. J. Am. Chem. Soc 2001;123:8628-8629. [PubMed: 11525684]

35. van Zijl PCM, Jones CK, Ren J, Malloy CR, Sherry AD. MRI detection of glycogen in vivo by using chemical exchange saturation transfer imaging (glycoCEST). Proc. Natl. Acad. Sci. USA 2007;104:4359-4364. [PubMed: 17360529]

36. McMahon MT, Gilad AA, Zhou J, Sun PZ, Bulte JWM, van Zijl PCM. Quantifying exchange rates in chemical exchange saturation transfer agents using the saturation time and saturation power dependencies of the magnetization transfer effects on the magnetic resonance imaging signal (QUEST and QUESP): $\mathrm{pH}$ calibration for poly-1-lysine and a starburst dendrimer. Magn. Reson. Med 2006;55:836-847. [PubMed: 16506187]

37. Jones CK, Schlosser MJ, van Zijl PCM, Pomper MG, Golay X, Zhou J. Amide proton transfer imaging of human brain tumors at 3T. Magn. Reson. Med 2006;56:585-592. [PubMed: 16892186]

38. Zhou J, Payen J-F, Wilson DA, Traystman RJ, van Zijl PCM. Using the amide proton signals of intracellular proteins and peptides to detect pH effects in MRI. Nat. Med 2003;9:1085-1090. [PubMed: 12872167]

39. Schroeder L, Lowery TJ, Hilty C, Wemmer DE, Pines A. Molecular imaging using a targeted magnetic resonance hyperpolarized biosensor. Science 2006;314:446-449. [PubMed: 17053143]

40. Oros AM, Shah NJ. Hyperpolarized xenon in NMR and MRI. Phys. Med. Biol 2004;49:R105-R153. [PubMed: 15566166]

41. Bouchiat MA, Carver TR, Varnum CM. Nuclear polarization in ${ }^{3} \mathrm{He}$ gas induced by optical pumping and dipolar exchange. Phys. Rev. Lett 1960;5:373-375.

42. Ward KM, Aletras AH, Balaban RS. A new class of contrast agents for MRI based on proton chemical exchange dependent saturation transfer (CEST). J. Magn. Reson 2000;143:79-87.87 [PubMed: 10698648] Introduced the idea of using exogenous compounds as CEST agents for MRI.

43. Ward KM, Balaban RS. Determination of $\mathrm{pH}$ using water protons and chemical exchange dependent saturation transfer (CEST). Magn. Reson. Med 2000;44:799-802. [PubMed: 11064415]

44. Powell DH, Ni Dhubhghaill OM, Pubanz D, Helm L, Lebedev YS, et al. High-pressure NMR kinetics. Part 74. Structural and dynamic parameters obtained from ${ }^{17} \mathrm{O}$ NMR, EPR, and NMRD studies of 
monomeric and dimeric $\mathrm{Gd}^{3+}$ complexes of interest in magnetic resonance imaging: an integrated and theoretically self-consistent approach. J. Am. Chem. Soc 1996;118:9333-9346.

45. Aime S, Barge A, Botta M, De Sousa AS, Parker D. Direct NMR spectroscopic observation of a lanthanide-coordinated water molecule whose exchange rate is dependent on the conformation of the complexes. Angew. Chem. Int. Ed 1998;37:2673-2675.2675 This important observation opened the way for the development of slow water-exchange agents as imaging agents.

46. Aime S, Barge A, Botta M, Parker D, De Sousa AS. Prototropic vs whole water exchange contributions to the solvent relaxation enhancement in the aqueous solution of a cationic $\mathrm{Gd}^{3+}$ macrocyclic complex. J. Am. Chem. Soc 1997;119:4767-4768.

47. Aime S, Barge A, Bruce JI, Botta M, Howard JAK, et al. NMR, relaxometric, and structural studies of the hydration and exchange dynamics of cationic lanthanide complexes of macrocyclic tetraamide ligands. J. Am. Chem. Soc 1999;121:5762-5771.

48. Zhang S, Winter P, Wu K, Sherry AD. A novel europium(III)-based MRI contrast agent. J. Am. Chem. Soc 2001;123:1517-1518.1518 [PubMed: 11456734] The first demonstration of a paramagnetic CEST agent as a MRI agent.

49. Zhang S, Wu K, Biewer MC, Sherry AD. ${ }^{1} \mathrm{H}$ and ${ }^{17} \mathrm{O}$ NMR detection of a lanthanide-bound water molecule at ambient temperatures in pure water as solvent. Inorg. Chem 2001;40:4284-4290. [PubMed: 11487334]

50. Bleaney B. Nuclear magnetic resonance shifts in solution due to lanthanide ions. J. Magn. Reson 1972;8:91-100.

51. Bleaney B, Dobson CM, Levine BA, Martin RB, Williams RJP, Xavier AV. Origin of lanthanide nuclear magnetic resonance shifts and their uses. J. Chem. Soc. Chem. Commun 1972;1972:791793.

52. Mironov VS, Galyametdinov YG, Ceulemans A, Gorller-Walrand C, Binnemans K. Roomtemperature magnetic anisotropy of lanthanide complexes. A model study for various coordination polyhedra. J. Chem. Phys 2002;116:4673-4685.

53. Zhang S, Merritt M, Woessner DE, Lenkinski RE, Sherry AD. PARACEST agents: modulating MRI contrast via water proton exchange. Acc. Chem. Res 2003;36:783-790.790 [PubMed: 14567712] Introduced the term PARACEST to describe paramagnetic CEST agents.

54. Zhang S, Wu K, Sherry AD. Unusually sharp dependence of water exchange rate versus lanthanide ionic radii for a series of tetraamide complexes. J. Am. Chem. Soc 2002;124:4226-4227. [PubMed: 11960448]

55. Woods M, Woessner DE, Zhao P, Pasha A, Yang M-Y, et al. Europium(III) macrocyclic complexes with alcohol pendant groups as chemical exchange saturation transfer agents. J. Am. Chem. Soc 2006;128:10155-10162. [PubMed: 16881645]

56. Zhang S, Michaudet L, Burgess S, Sherry AD. The amide protons of an ytterbium(III) dota tetraamide complex act as efficient antennae for transfer of magnetization to bulk water. Angew. Chem. Int. Ed 2002;41:1919-1921.

57. Terreno E, Castelli DD, Cravotto G, Milone L, Aime S. Ln(III)-DOTAMGly complexes: a versatile series to assess the determinants of the efficacy of paramagnetic chemical exchange saturation transfer agents for magnetic resonance imaging applications. Investig. Radiol 2004;39:235-243. [PubMed: 15021328]

58. Aime S, Barge A, Castelli DD, Fedeli F, Mortillaro A, et al. Paramagnetic Lanthanide(III) complexes as $\mathrm{pH}$-sensitive chemical exchange saturation transfer (CEST) contrast agents for MRI applications. Magn. Reson. Med 2002;47:639-648. [PubMed: 11948724]

59. Yoo B, Pagel MD. Peptidyl molecular imaging contrast agents using a new solid-phase peptide synthesis approach. Bioconjug. Chem 2007;18:903-911. [PubMed: 17330953]

60. Yoo B, Pagel MD. A PARACEST MRI contrast agent to detect enzyme activity. J. Am. Chem. Soc 2006;128:14032-14033. [PubMed: 17061878]

61. Zhang S, Malloy CR, Sherry AD. MRI thermometry based on PARACEST agents. J. Am. Chem. Soc 2005;127:17572-17573. [PubMed: 16351064]

62. Wegh, RT.; Pikkemaat, JA.; Willard, NP., inventors. WO Patent No. 2006-IB51237. 2006114739. 2006. 
63. Willard, NP.; Wegh, RT.; Pikkemaat, JA.; Gruell, H., inventors. WO Patent No. 2006-IB51235. 2006114738. 2006. Pikkemaat JA, Wegh RT, Lamerichs R, van de Molengraaf RA, Langereis S, et al. Dendritic PARACEST contrast agents for magnetic resonance imaging. Contrast Media Mol. Imaging 2007;2:229-239. [PubMed: 17937448]

64. Trokowski R, Zhang S, Sherry AD. Cyclen-based phenylboronate ligands and their $\mathrm{Eu}^{3+}$ complexes for sensing glucose by MRI. Bioconjug. Chem 2004;15:1431-1440. [PubMed: 15546212]

65. Zhang S, Trokowski R, Sherry AD. A paramagnetic CEST agent for imaging glucose by MRI. J. Am. Chem. Soc 2003;125:15288-15289. [PubMed: 14664562]

66. Trokowski R, Ren JM, Kalman FK, Sherry AD. Selective sensing of zinc ions with a PARACEST contrast agent. Angew. Chem. Int. Ed 2005;44:6920-6923.

67. Micskei K, Helm L, Brucher E, Merbach AE. Oxygen-17 NMR study of water exchange on gadolinium polyaminopolyacetates $\left[\mathrm{Gd}(\mathrm{DTPA})\left(\mathrm{H}_{2} \mathrm{O}\right)\right]^{2-}$ and $\left[\mathrm{Gd}(\mathrm{DOTA})\left(\mathrm{H}_{2} \mathrm{O}\right)\right]^{-}$related to NMR imaging. Inorg. Chem 1993;32:3844-3850.

68. Ratnakar J, Woods M, Lubag AJM, Kovacs Z, Sherry AD. Modulation of water exchange in europium (III) DOTA-tetraamide complexes via electronic substituent effects. J. Am. Chem. Soc 2008;130:67. [PubMed: 18067296]

69. Aime S, Botta M, Fasano M, Crich SG, Terreno E. Gd(III) complexes as contrast agents for magnetic resonance imaging: a proton relaxation enhancement study of the interaction with human serum albumin. J. Biol. Inorg. Chem 1996;1:312-319.

70. Aime S, Chiaussa M, Digilio G, Gianolio E, Terreno E. Contrast agents for magnetic resonance angiographic applications: ${ }^{1} \mathrm{H}$ and ${ }^{17} \mathrm{O}$ NMR relaxometric investigations on two gadolinium(III) DTPA-like chelates endowed with high binding affinity to human serum albumin. J. Biol. Inorg. Chem 1999;4:766-774. [PubMed: 10631608]

71. Hovland R, Aasen AJ, Klaveness J. Preparation and in vitro evaluation of GdDOTA-(BOM) 4 ; a novel angiographic MRI contrast agent. Org. Biomol. Chem 2003;1:1707-1710. [PubMed: 12926358]

72. Ali M, Woods M, Suh E, Kovacs Z, Tircso' G, et al. Albumin-binding PARACEST agents. J. Biol. Inorg. Chem 2007;12:855-865. [PubMed: 17534672]

73. Caravan P, Cloutier NJ, Greenfield MT, McDermid SA, Dunham SU, et al. The interaction of MS-325 with human serum albumin and its effect on proton relaxation rates. J. Am. Chem. Soc 2002;124:3152-3162. [PubMed: 11902904]

74. Zech SG, Eldredge HB, Lowe MP, Caravan P. Protein binding to lanthanide(III) complexes can reduce the water exchange rate at the lanthanide. Inorg. Chem 2007;46:3576-3584. [PubMed: 17425306]

75. Winter PM, Cai KJ, Chen J, Adair CR, Kiefer GE, et al. Targeted PARACEST nanoparticle contrast agent for the detection of fibrin. Magn. Reson. Med 2006;56:1384-1388. [PubMed: 17089356]

76. Winter PM, Caruthers SD, Kassner A, Harris TD, Chinen LK, et al. Molecular imaging of angiogenesis in nascent vx-2 rabbit tumors using a novel $\mathrm{a}_{\mathrm{n}} \mathrm{b}_{3}$-targeted nanoparticle and 1.5 Tesla magnetic resonance imaging. Cancer Res 2003;63:5838-5843. [PubMed: 14522907]

77. Flacke S, Fischer S, Scott MJ, Fuhrhop RJ, Allen JS, et al. Novel MRI contrast agent for molecular imaging of fibrin implications for detecting vulnerable plaques. Circulation 2001;104:1280-1285. [PubMed: 11551880]

78. Adair C, Woods M, Zhao PY, Pasha A, Winters PM, et al. Spectral properties of a bifunctional PARACEST europium chelate: an intermediate for targeted imaging applications. Contrast Media Mol. Imaging 2007;2:55-58. [PubMed: 17326038]

79. Schmieder AH, Winter PM, Caruthers SD, Harris TD, Williams TA, et al. Molecular MR imaging of melanoma angiogenesis with $\mathrm{a}_{\mathrm{n}} \mathrm{b}_{3}$-targeted paramagnetic nanoparticles. Magn. Reson. Med 2005;53:621-627. [PubMed: 15723405]

80. Aime S, Castelli DD, Terreno E. Highly sensitive MRI chemical exchange saturation transfer agents using liposomes. Angew. Chem. Int. Ed 2005;44:5513-5515.

81. Aime S, Castelli DD, Lawson D, Terreno E. Gd-loaded liposomes as $\mathrm{T}_{1}$, susceptibility, and CEST agents, all in one. J. Am. Chem. Soc 2007;129:2430-2431. [PubMed: 17288421] 

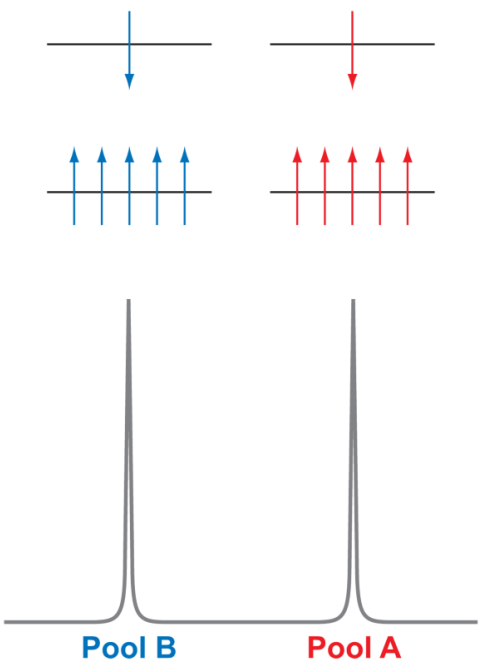
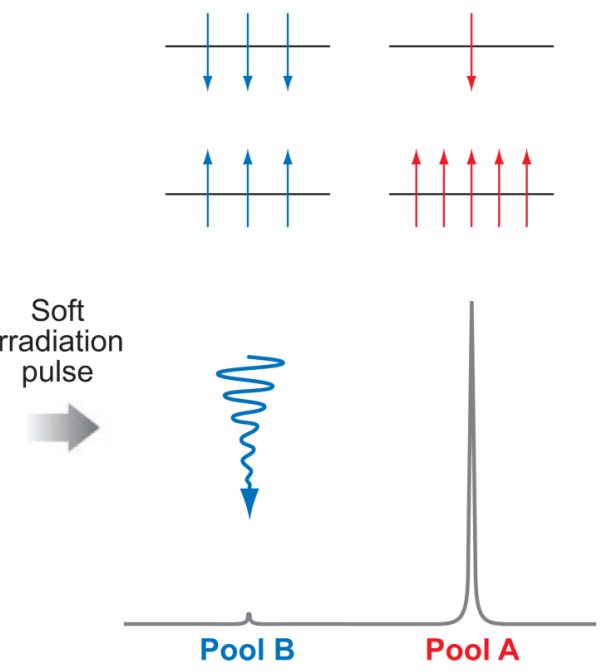
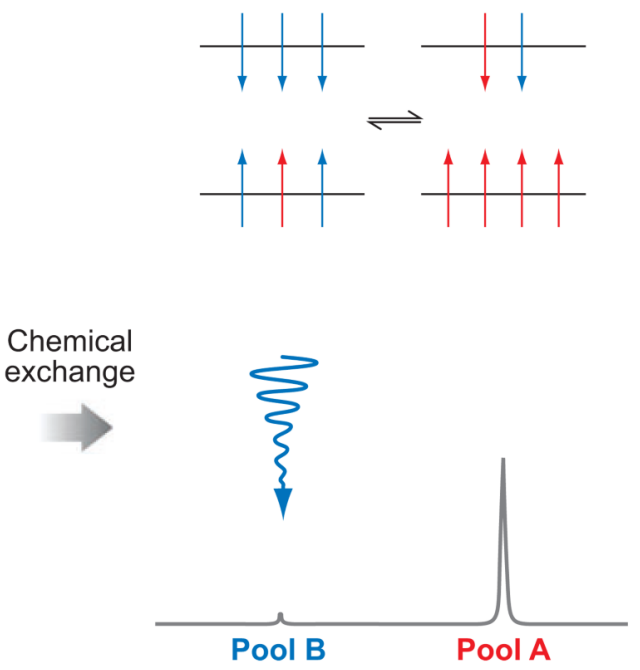

Figure 1.

Schematic representations of the distribution of spins, aligned with and against the field (upper and lower energy levels, respectively) (above) and simulated NMR spectra (below) for two chemically distinct pools of nuclei (left), two spins after a saturation pulse has been applied to one pool (middle), and for a system undergoing chemical exchange after a saturation pulse has been applied to one pool (right). 

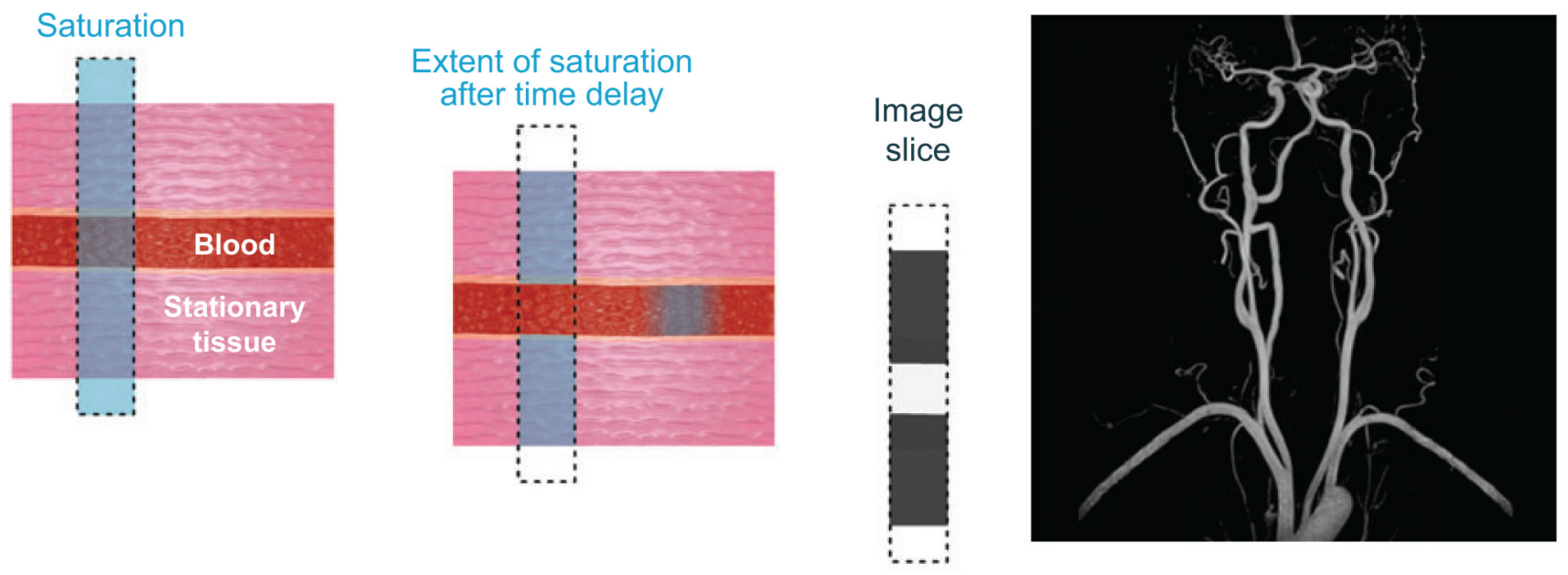

Figure 2.

Angiography by TOF-MRA is a form of saturation transfer experiment. An image slice, represented by the dotted line, is acquired by saturating the bulk water protons only in that image slice (left, the saturation is represented in blue). After a short time delay, the saturation of water protons in blood has been transferred out of the slice and replaced by unsaturated spins (middle). Thus, when the image is acquired, the stationary tissue appears dark but the blood is bright. In this way, angiograms may be acquired, such as the one of the carotid arteries shown (right). 

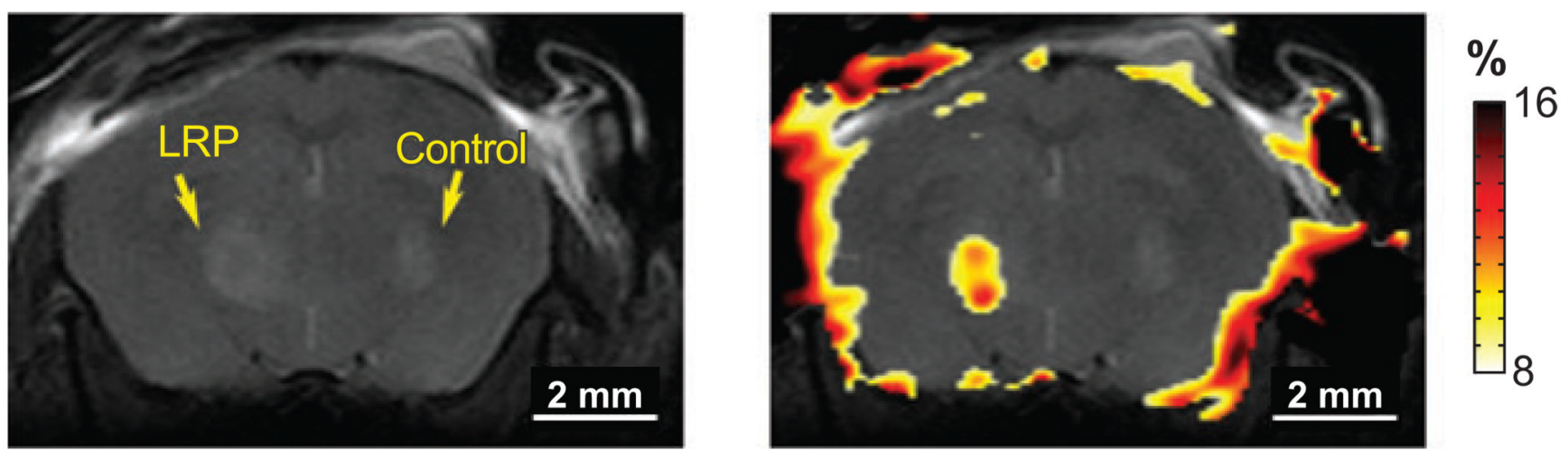

Figure 3.

An image of a mouse brain implanted with glioma cells expressing LRP (left hemisphere) and glioma cells (right hemisphere). On the left is an anatomical image, on the right a colorized difference image of the areas that exhibit CEST has been overlaid on the anatomical image. The LRP gives rise to a significant change in water signal intensity, allowing the genetically modified cells to be easily identified and tracked by CEST imaging. The area of CEST intensity around the skull is thought to be due to field inhomogeneities. Images reproduced, with permission, from Nature Biotechnology. 

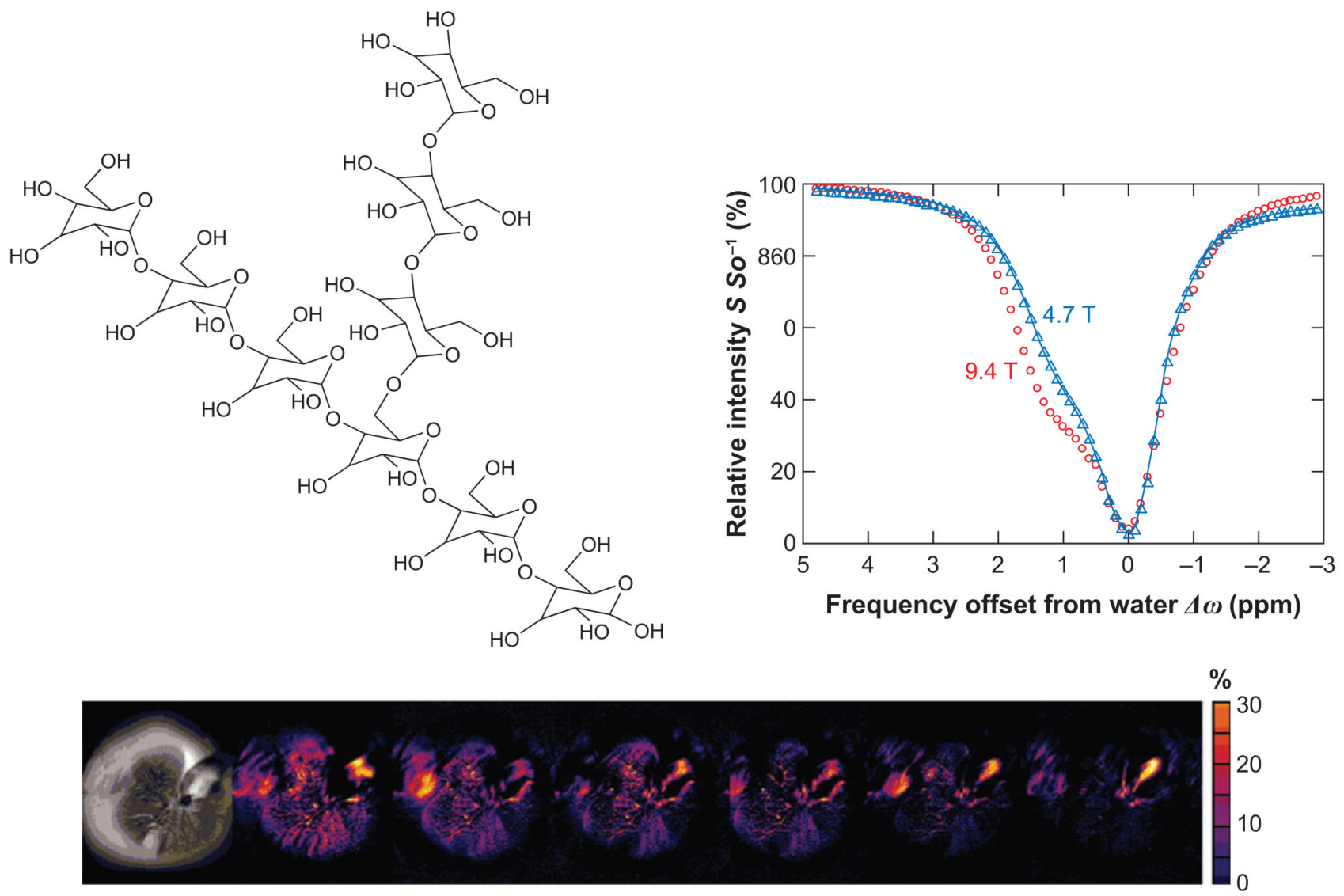

Figure 4.

The structure of glycogen (top left) and its CEST spectra recorded at 200 and $400 \mathrm{MHz}$ (top right). An anatomical image, with a presaturation pulse applied at $+1 \mathrm{ppm}$, shows a darkening of the image due to the presence of glycogen (bottom left). Colorized CEST difference images acquired at $0,20,40,60,80,100$, and $120 \mathrm{~min}$ after administration of glucagon become progressively less orange, reflecting depletion of glycogen (bottom). MR images and CEST spectra reproduced from Proceedings of the National Academy of Sciences. 


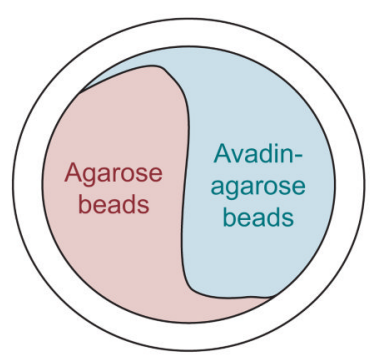

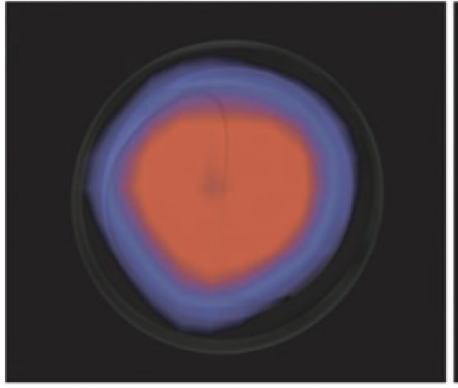

Bead signal HYPER-CEST off

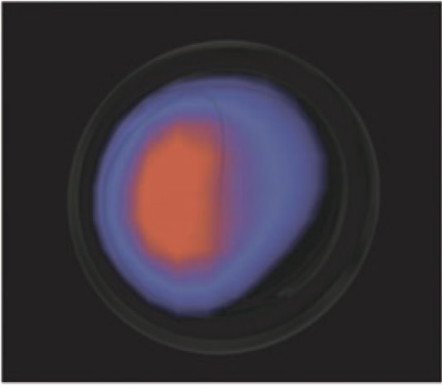

Bead signal HYPER-CEST on

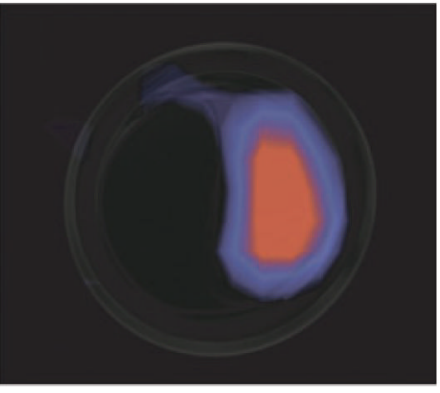

Bead signal difference

= biosensor image

Figure 5.

The setup of the phantom system used to image the CEST properties of hyperpolarized ${ }^{129} \mathrm{Xe}$ (far left). Images of the phantom system showing the signal arising from free ${ }^{129} \mathrm{Xe}(l e f t)$ and free ${ }^{129} \mathrm{Xe}$ after presaturation of the encapsulated xenon (right). The difference image (far right) shows that the CEST effect is only generated for those regions in which the avadin target is found. MR images from Reference 39. Reprinted with permission from AAAS. 

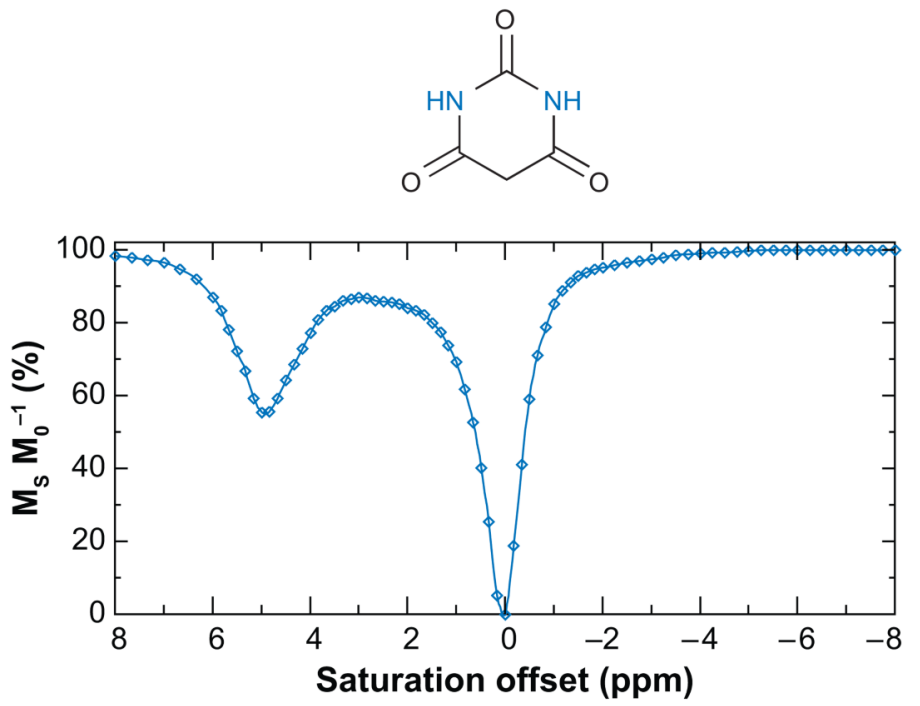
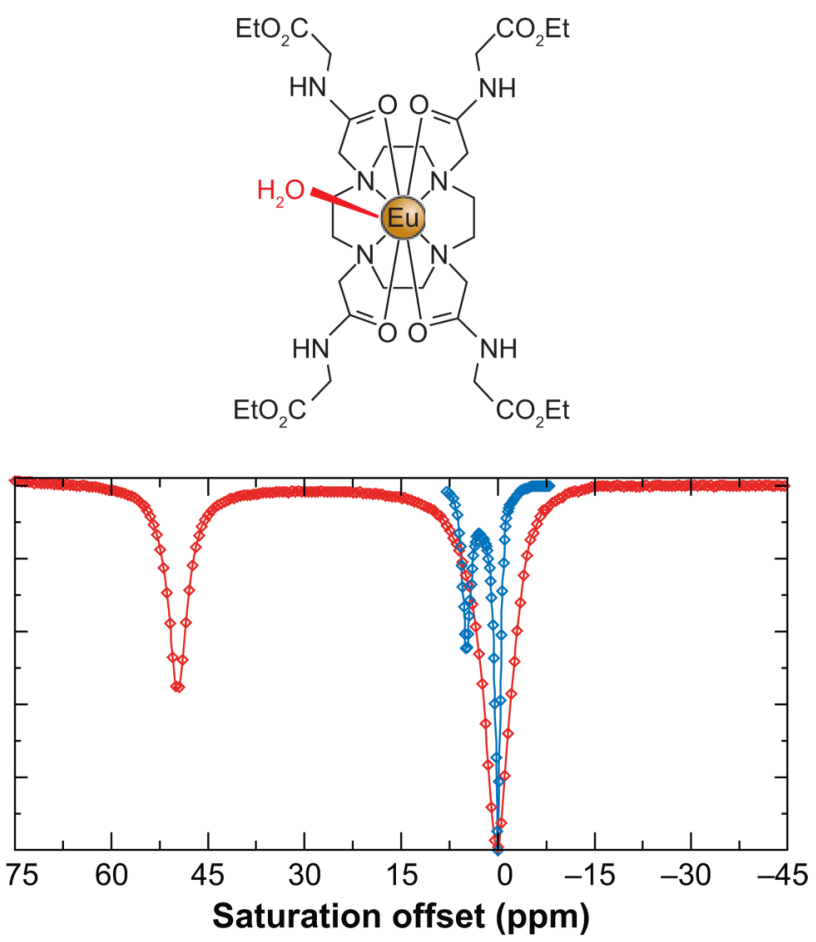

Figure 6.

The CEST spectra of barbituric acid (left) and a Eu ${ }^{3+}$ complex, EuDOTA-(glycine ethyl ester $)_{4}$ (right). The CEST spectrum of barbituric acid is shown on the same scale as that of the $\mathrm{Eu}^{3+}$ complex (blue, right) for comparative purposes. 

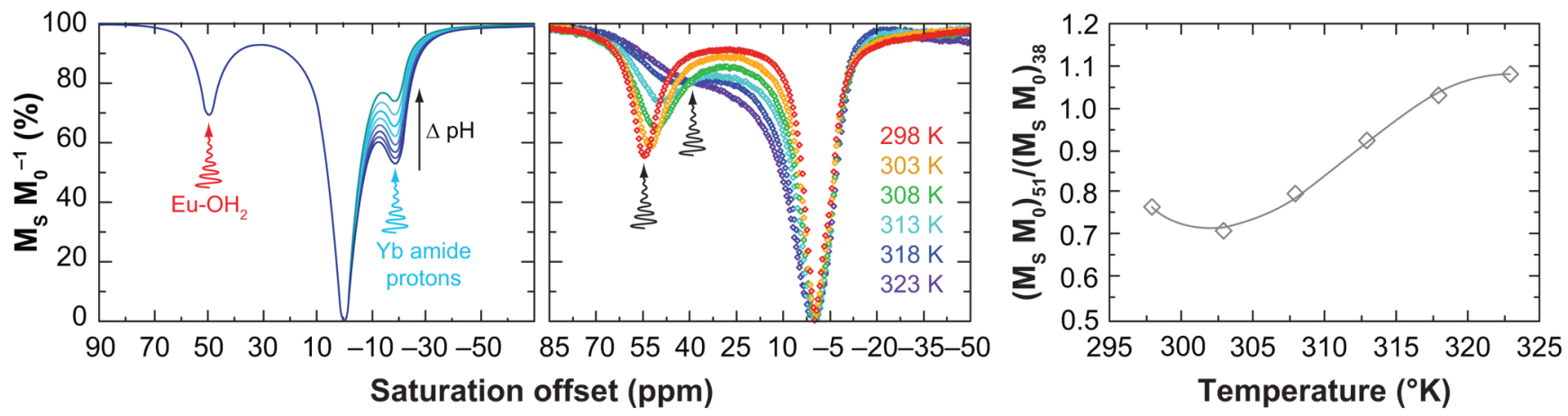

Figure 7.

CEST imaging allows ratiometric determination of biologically relevant parameters such as $\mathrm{pH}$ and temperature. (Left) simulated CEST spectra of a cocktail of $\mathrm{Eu}^{3+}$ and $\mathrm{Yb}^{3+}$ DOTAtetraamide complexes with varying $\mathrm{pH}$. The insensitivity of CEST from the metal-bound water of the $\mathrm{Eu}^{3+}$ complex can be used as a concentration marker, whereas the $\mathrm{pH}$-sensitive amide protons of the $\mathrm{Yb}^{3+}$ complexes can be used to measure pH. (Right) The CEST spectra of an $\mathrm{Eu}^{3+}$ DOTA-tetraamide complex at different temperatures. 

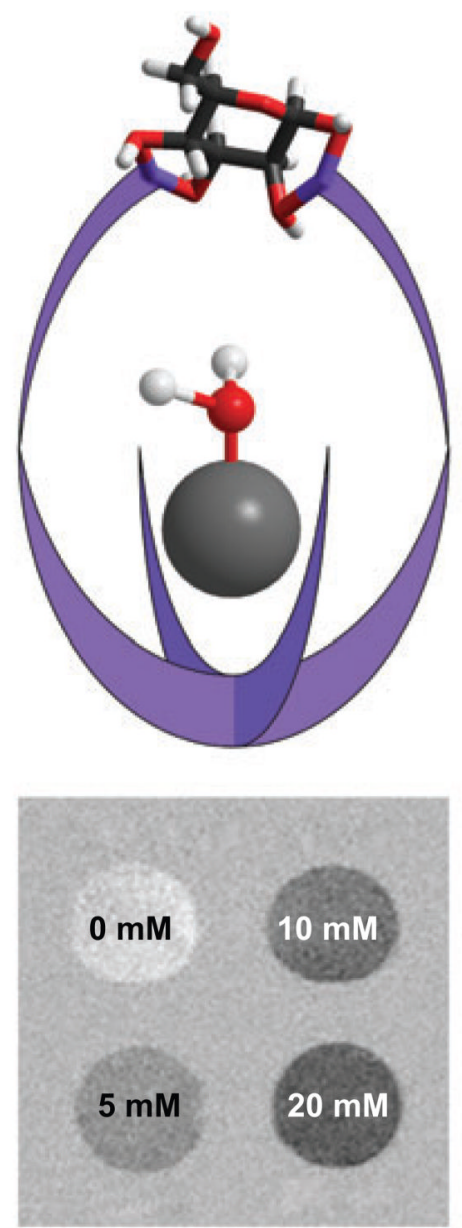
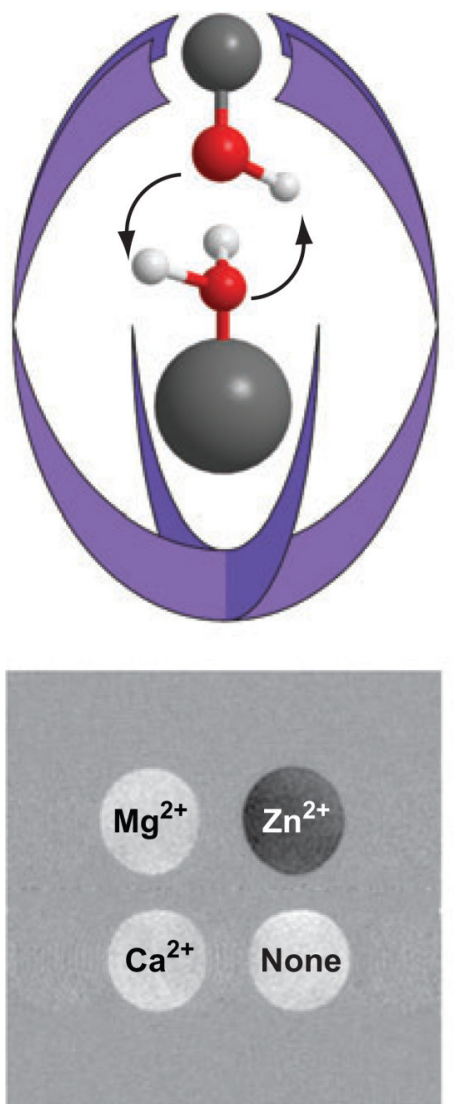
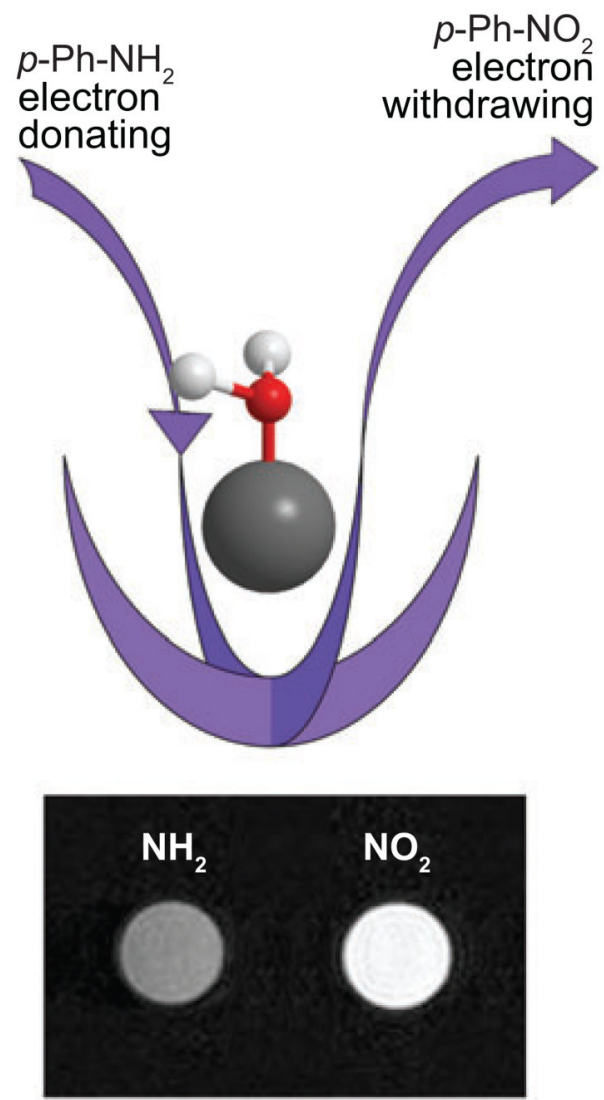

Figure 8.

Illustrating different methods of designing responsive PARACEST agents. (Left) The binding of glucose hinders water exchange, increasing CEST. (Middle) At physiological pH, binding of $\mathrm{Zn}^{2+}$ at its binding site above the $\mathrm{Eu}^{3+}-\mathrm{OH}_{2}$ molecule results in catalysis of proton exchange, altering the CEST properties. (Right) Altering the electronic effects of substituents can affect the interaction between the metal and water, which may be observed as a change in CEST. 


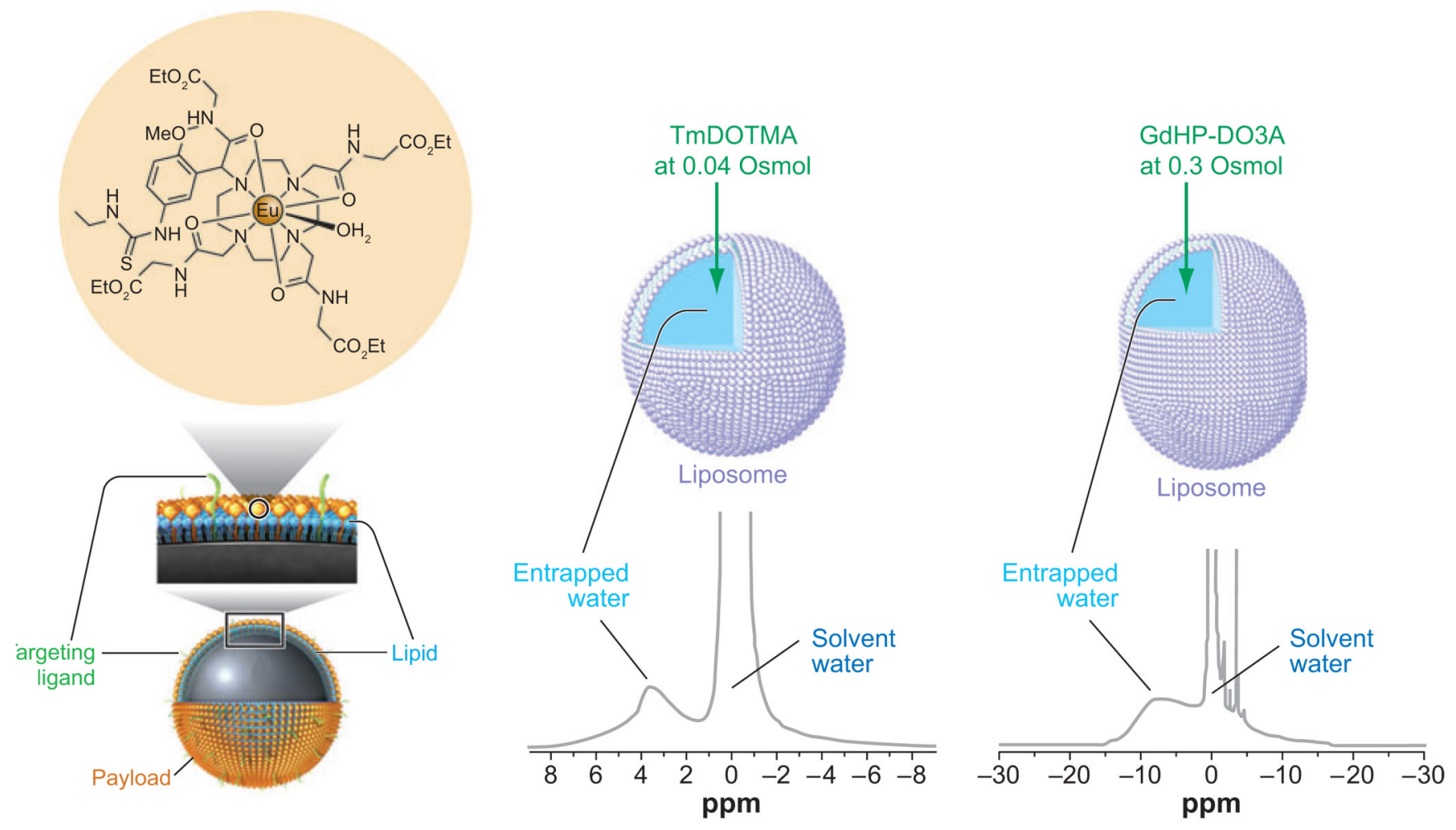

Figure 9.

Three examples of ways to enhance CEST sensitivity. (Left) A perfluorocarbon-filled nanoparticle has a large number of CEST agents incorporated onto the surface of the nanoparticle. (Middle) A spherical liposome is filled with a high concentration of TmDOTMA, a reagent that exchanges water rapidly and shifts the average position of the intraliposomal water downfield by nearly 4 ppm. (Right) An osmotically shrunk liposome filled with a high concentration of GdHP-DO3A, a clinically approved agent, results in a highly shifted intraliposomal water peak due to bulk susceptibility differences between the two water compartments. 


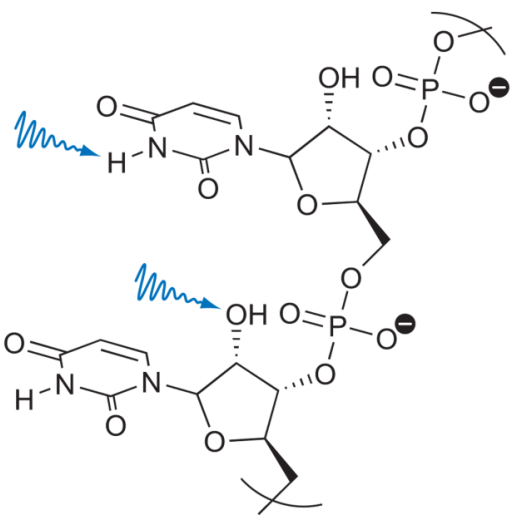
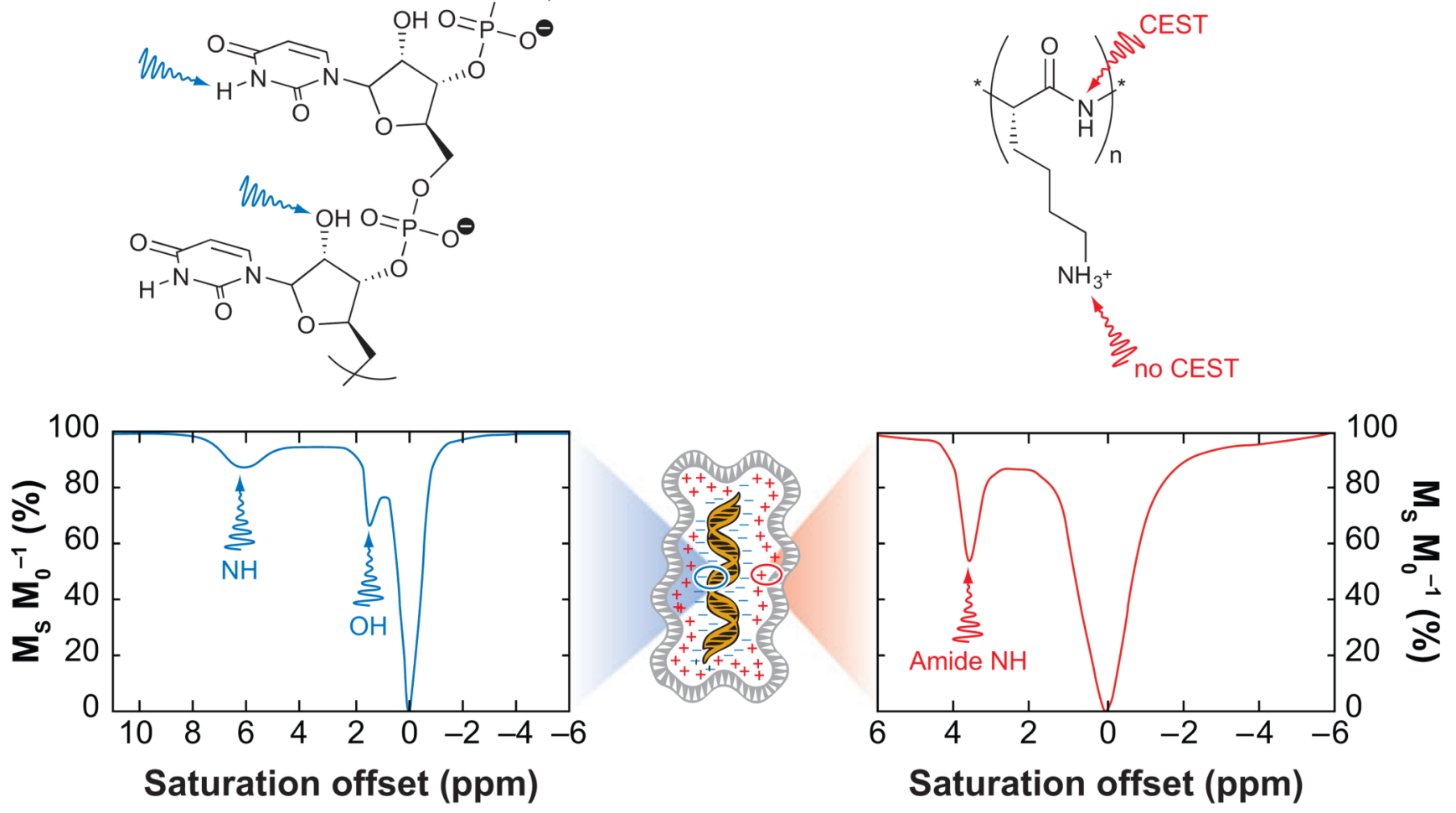

Figure 10.

A schematic representation of a gene therapy bundle of nucleic acids and cationic polymers. The RNA strand poly(rU) (left) has two sites that give rise to CEST. The amide protons in cationic polymers, such as PLL (right), can also give rise to a CEST effect. 